Article

\title{
Bumpless Transfer of Parametrized Multivariable Disturbance Observer Based Controller to Reduce Cyclic Loads of Wind Turbine
}

\author{
Raja M. Imran ${ }^{1 *}$, D. M. Akbar Hussain ${ }^{2}$, Bhawani Shanker Chowdhry ${ }^{3}$ and Arbab Waleed ${ }^{1}$ \\ Smart PCBs, Rawalpindi, Pakistan \\ Department of Energy Technology, Aalborg University, Esbjerg, Denmark \\ 3 DEAN Faculty of Electrical, Electronics Computer Engineering, Mehran University of Engineering \\ Technology, Jamshoro, Pakistan \\ * Correspondence: rmimran2001@gmail.com; Tel.: +92-345-5639-546
}

\begin{abstract}
This paper is concerned with bump-less transfer of parametrized disturbance observer based controller (DOBC) with Individual Pitch Control (IPC) strategy for full load operation of wind turbine. Aerodynamic cyclic loads are reduced by tuning multivariable DOBC with the objective to reduce output power fluctuation, tower oscillation and drive-train torsion. Furthermore tower shadow and wind shear effect are also mitigated using parametrized controller. A scheduling mechanism between two DOBC is developed and tested on Fatigue, Aerodynamics, Structures, and Turbulence ( FAST) code model of National Renewable Energy Laboratory (NREL)'s 5 MW wind turbine. The closed-loop system performance is assessed by comparing the simulation results of proposed controller with a fixed gain and Linear Parameter Varying (LPV) DOBC with Collective Pitch Control (CPC) for full load operation. It is tested with step changing wind to see the behavior of the system under step change with wind shear and tower shadow (cyclic load) effects. Also turbulent wind is applied to see the smooth transition of the controllers. It can be concluded from the results that the proposed parametrized control DOBC with IPC shows smooth transition from one controller to another by interpolation. Moreover fatigue of the gear and tower due to wind shear and tower shadow effects are reduced considerably by the proposed controller as compared to collective pitch control.
\end{abstract}

Keywords: Wind Turbine, LPV, DOBC, Multivariable

\section{Introduction}

The main objective for wind turbine controllers is to maximize the annual energy production and reduce the maintenance cost. Pitch-regulated variable-speed wind turbines has two operational modes: partial load and full load operations.In partial load operation, kinetic energy in the wind is not enough to achieve nominal electrical power output and the objective is to control the pitch and rotor speed to achieve the maximum aerodynamic efficiency of the wind turbine. In full load operation, kinetic energy in the wind increases resulting in increased nominal electrical power output and objective is to keep generator speed close to the nominal speed, and pitch angle is controlled to achieve nominal electrical power production. In both operating regions, it is important to reduce the fluctuations in the output power and minimize the fatigue loads of structural components like drive train, tower and pitch system with of cyclic loads. PID is used to regulate rotor speed by using pitch mechanism to reduce wind disturbance effect in Region III. An expert PID controller has been used [1] to restrain the overshoot of rotor speed and provide better performance with varying parameters 
of the controller. Modern Control systems are based on state space model and have capability to handle MIMO system in an efficient manner. Linear Quadratic Gaussian (LQG) is used for the power regulation in high wind speed [2][3] for pitch regulated

variable speed wind turbines using collective pitch control; result showed that the LQG controller has good power regulation as compared to PI control. LQG controller [4] has been developed to reduce loads on wind turbine component and regulate power in the presence of measurement noise using for pitch control. Torque control of Doubly Fed Induction Generator( DFIG) [5] is used to reduce flicker in the output power and [6] implemented an individual pitch control (IPC) strategy to mitigate the fluctuation in output power caused by wind shear and tower shadow. DAC is used to model and simulate system with known disturbance waveform. DAC has been used [7] to mitigate the effect of disturbances by using collective pitch control of wind turbine. In [8] multivariable DAC is developed to mitigate the effect of periodic loads (wind shear and tower shadow) with multiple objectives using pitch control of wind turbine. Multivariable control algorithm used [9] [10] PI control for regulating generator speed and independent pitch control (IPC) to reduce structural loads. Nonlinear state feedback torque control is used [11] for the above-rated power operating condition of wind turbine.$H_{\infty}$ norm minimization for collective and cyclic pitch has been used [12] to increase damping of the first axial tower bending mode and reduced $1 \mathrm{p}$ fluctuations in blade root bending moments. Observer-based output feedback control for uncertain systems [13] is used to minimize the energy while variable structure control law is used for robustness to uncertainty and Disturbance-observer based controllers (DOBC) are reviewed [14].

Ostergaard et al. observed that the operation of wind turbines at different wind speeds require some kind of gain scheduling, so they have applied Linear Parameter Varying control (LPV) to develop robust controllers that cater for a both partial load and full load conditions [15]. Gain scheduling strategy [16] used for multivariable controller which interpolates between unstable controllers based on $\mu$ synthesis. One important step is the gain scheduling of linear controllers such that the controller coefficients are scheduled with the current value of the exogenous or endogenous scheduling signal. A safe bumpless transfer has been proposed [17] between two observer-based controllers for linear multivariable system by the interpolation of covariance to keep the closed loop system stable and bumpless transfer with integral action based on the Youla-Kucera parametrization is used for wind turbine [18]. LPV design methods are investigated for wind turbine [19] and gain-scheduling (GS) control techniques to floating offshore wind turbines on barge platforms is presented. Modeling and controller performance evaluation are presented [20][21][22] for both low and high wind speed cases. Multivariable control techniques are used to reduce the fatigue of wind turbine components with CPC and IPC. LQG controller is developed to mitigate the effect of sensor noise[4], DAC with optimal control theory is designed to get better stability of output power[7] and 3p harmonics generated due to periodic loads are reduced using CPC [8][25]. This paper presents a systematic approach to design a LPV-DOBC with IPC (LPV-IPC) for full load operation of wind turbine with multiple objectives. Bumpless transfer between controllers is accomplished by interpolation of covariance of linear DOBC controllers with CPC and parametrized controller is tested on FASTCode [23] model of NREL's 5MW wind turbine with actuator dynamics. The closed loop performance is evaluated by simulation of fixed gain DOBC with CPC (DOBC-CPC), fixed gain DOBC with IPC (DOBC-IPC) and the LPV-DOBC-IPC. Simulation are performed with step changing and turbulent wind to see the bumpless transfer between the multivariable Linear Time Invariant (LTI) controllers in full load operation of wind turbine. Organization of the paper is that Section 2 describes the DOBC, Section 3 is about the bumpless transfer method. Section 4 shows simulation results and conclusions are given in Section 5. 


\section{Disturbance Observer Based Control (DOBC)}

State space model of wind turbine is

$$
\begin{gathered}
\dot{x}=A x(t)+B u(t)+B_{d} u_{d}(t) \\
y(t)=C x(t)+D u(t)
\end{gathered}
$$

$A, B, B_{d}, C, D$ are state transition, control input, disturbance input, measured state and output matrices of the plant respectively. $u$ is the input, $x$ is state and $y$ is the output vector of the system.

Disturbance waveform [7][8] can be represented as

$$
\begin{gathered}
u_{d}(t)=\theta z_{d}(t) \\
\dot{z}_{d}(t)=F z_{d}(t) ; \quad \dot{z}_{d}(0)=z_{d}^{0}
\end{gathered}
$$

where $z_{d}$ is state of the disturbance, $u_{d}$ is for disturbance. $z_{d}^{0}$ is the initial state of the disturbance. $F$ is the state transition matrix and $\theta$ is the output matrix of the disturbance waveform. Kalman estimator is used to estimate the states of the plant as

$$
\begin{gathered}
\dot{\hat{x}}(t)=A \hat{x}(t)+B u(t)+B_{d} \hat{u}_{d}(t)+L_{x}(y(t)-\hat{y}(t)) \\
\hat{y}(t)=C \hat{x}(t) ; \hat{x}(0)=0
\end{gathered}
$$

$\hat{x}$ is estimated state of the plant and $\hat{z}_{d}$ is estimated state of the disturbance, $\hat{u}, \hat{u}_{d}$ and $\hat{y}$ are estimated input, disturbance and output respectively. It is also used to estimate the state of the disturbance as

$$
\begin{gathered}
\dot{\hat{z}}_{d}=F \hat{z}_{d}(t)+L_{d}(y(t)-\hat{y}(t)) \\
\hat{u}_{d}(t)=\theta \hat{z}_{d}(t) ; \quad \hat{z}_{d}(0)=0
\end{gathered}
$$

To mitigate the effect of disturbance, control law is

$$
u=-K_{x} \hat{x}(t)-K_{d} \hat{z}_{d}(t)
$$

s8 $L_{x}$ is the plant and $L_{d}$ is the disturbance state estimation matrix, $K_{x}$ is full state feedback 89 matrix and is calculated by using optimal control theory [27][28]. $K_{d}$ is disturbance feedback matrix 9o calculated independently [7] to make closed loop system "disturbance free".

91 DOBC-CPC [24]can be represented as

$$
K(s)=\left[\begin{array}{cc|c}
A-B K_{x}-L_{x} C+L_{x} D K_{x} & B_{d} \theta-B K_{d}+L_{x} D K_{d} & L_{x} \\
L_{d} D K_{x}-L_{d} C & L_{d} D K_{d}+F & L_{d} \\
\hline-K_{x} & -K_{d} & 0
\end{array}\right]
$$

93 DOBC-IPC [25] can be written as 


$$
K(s)=\left[\begin{array}{cc|c}
A-B K_{x}-L_{x} C+L_{x} D K_{x} & B_{d} \theta_{d}-B K_{d}+L_{x} D K_{d} & L_{x} \\
L_{d} D K_{x}-L_{d} C & L_{d} D K_{d}+F & L_{d} \\
\hline 0 & -K_{d p} & 0 \\
-K_{x} & -K_{d s} & 0
\end{array}\right]
$$

94 Where $K_{d p}$ is the disturbance feedback to mitigate the periodic disturbance and $K_{d s}$ is for step 95 mitigation. Disturbance feedback is the sum of periodic and step feedback matrices.

\section{3. Bumpless Transfer Method for DOBC}

Let $G_{0}(s)$ and $G_{1}(s)$ be the detectable and stablizable linearized plant of $G(s)$ at two operating points and is scheduled [16] as

$$
G_{\gamma}(s)=(1-\gamma) G_{o}(s)-\gamma G_{1}(s)
$$
disturbance feedback matrices respectively for the second controller.

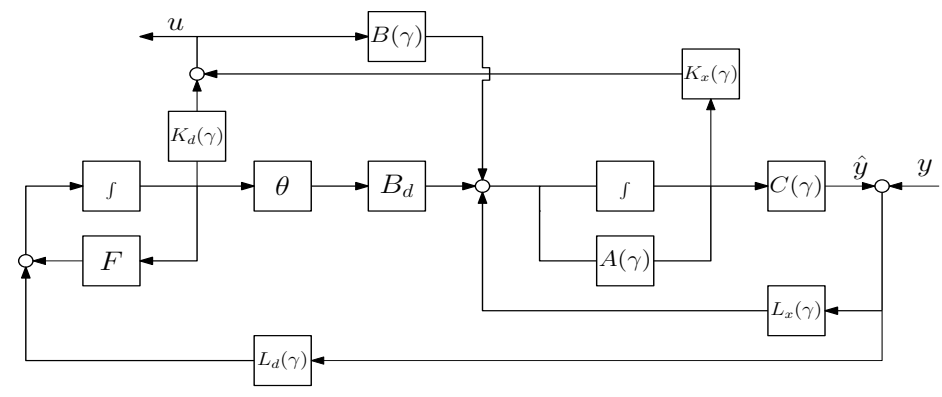

Figure 1. Parametrized DOBC Structure

$$
\begin{aligned}
& K_{d}(\gamma)=(1-\gamma) K_{d 0}-\gamma K_{d 1} \\
& K_{x}(\gamma)=(1-\gamma) K_{x 0}-\gamma K_{x 1} \\
& L_{d}(\gamma)=(1-\gamma) L_{d 0}-\gamma L_{d 1} \\
& L_{x}(\gamma)=(1-\gamma) L_{x 0}-\gamma L_{x 1}
\end{aligned}
$$

Then $K_{\gamma}(s)$ is the family of internally stable disturbance accommodated observer based controllers [17] can be represented as

$$
K_{\gamma}(s)=\left[\begin{array}{cc|c}
A_{11}(\gamma) & A_{12}(\gamma) & L_{x} \\
A_{21}(\gamma) & A_{22}(\gamma) & L_{d} \\
\hline-K_{x} & -K_{d} & 0
\end{array}\right]
$$

Where

$$
\begin{gathered}
A_{11}(\gamma)=A(\gamma)-B(\gamma) K_{x}(\gamma)-L_{x}(\gamma) C(\gamma)+L_{x}(\gamma) D(\gamma) K_{x}(\gamma) \\
A_{12}(\gamma)=B_{d}(\gamma) \theta-B(\gamma) K_{d}(\gamma)+L_{x}(\gamma) D(\gamma) K_{d}(\gamma) \\
A_{21}(\gamma)=L_{d}(\gamma) D(\gamma) K_{x}(\gamma)-L_{d}(\gamma) C(\gamma)
\end{gathered}
$$




$$
A_{21}(\gamma)=L_{d}(\gamma) D(\gamma) K_{d}(\gamma)+F
$$

$\gamma \epsilon(0,1)$ is the scheduling parameter, $\hat{v}$ is the estimated rotor wind speed [26] which is used for the scheduling of parametrized controller.

$A(\gamma), B(\gamma), B_{d}(\gamma), C(\gamma), D(\gamma)$ are state transition, control input, disturbance input, measured state and output matrices of the interpolated plant $G_{\gamma}(s)$ between the operating points respectively.

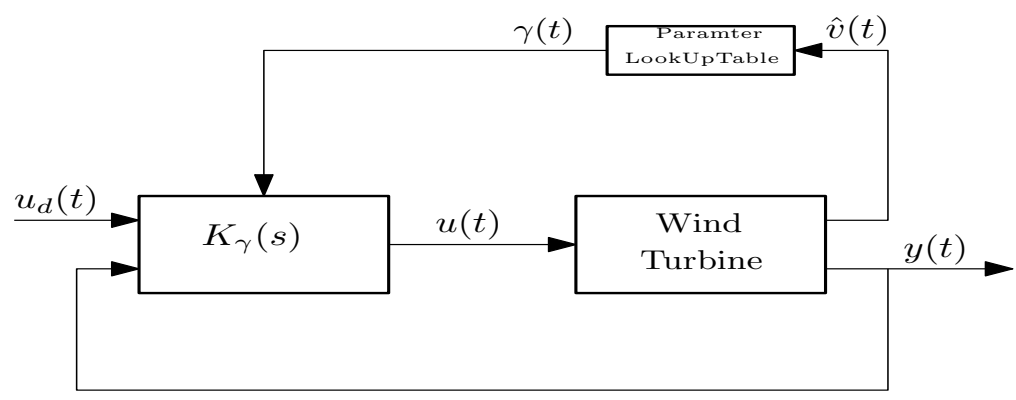

Figure 2. Closed loop System

\section{Wind Turbine Control Simulation}

The proposed control is tested on National Renewable Energy Laboratory (NREL)'s 5MW [29] wind turbine model. State feedback and estimator gain matrices are chosen using optimal control theory for fixed gain DOBC [8][7] at the operating points to reduce fatigue of structural components due to wind shear and tower shadow effects using CPC. The same parameters are then used for the tuning of DOBC with IBP and a scheduling method is described for smooth transition from one controller to another controller. Operating points are chosen [15] at mid wind speed for full load operation with rated generator torque as $(18 \mathrm{~m} / \mathrm{s}, 14.92 \mathrm{deg})$ and $(19 \mathrm{~m} / \mathrm{s}, 16.23 \mathrm{deg})$. Wind speed used for the scheduling of controller is estimated [26] and controllers between the operating points are interpolated as illustrated in Figure 1. Closed loop system for wind turbine with actuator dynamics and parametrized controller is shown in Figure 2. The proposed controller performance is evaluated by applying step changing wind to see the behavior at the operating point and then turbulent wind generated with mean of mid wind speed for full load operation of wind turbine.

The closed-loop performance is assessed by simulations of the proposed LPC-IPC with fixed gain-CPC and LPV-CPC. The performance is analyzed by measuring the fluctuation in gen speed, mitigation of fatigue of drive train, reduction in pitching activity and bumpless transfer between the controller of wind turbine. Results from simulation in full load are given in Figure 3 in which the most important observation is that the generator speed variations, structural loads and pitching activity are reduced by the proposed controller as compared to other two controllers.

For Step analysis of the system, a step changing wind is applied to the wind turbine with wind shear and tower shadow effect. From the comparison of the results in Figure fig-3, it can seen that LPV-IPC (proposed controller) has less fluctuation in the generator speed at step change and the periodic loads are well mitigated as compared to fixed gain-CPC and LPV-CPC. Drive-train torsion and pitching activity is reduced for step with wind shear and tower shadow effects.

Finally, the scheduling of the proposed controller is tested by performing a simulation with turbulent wind with mean of $18 \mathrm{~m} / \mathrm{s}$ and turbulence value of 10 generated from turbSim for above rated wind speed condition. The purpose of this simulation is to investigate the controller transitions along the operating trajectory. Such a simulation is given in Figure 4 from which it can be seen that the controller provides a glitch free transfer of controller for above rated wind speed condition. Also fatigue of drive-train is reduced, less pitching activity and better power regulation as compared to fixed gain and LPV DOBC controllers with CPC. 

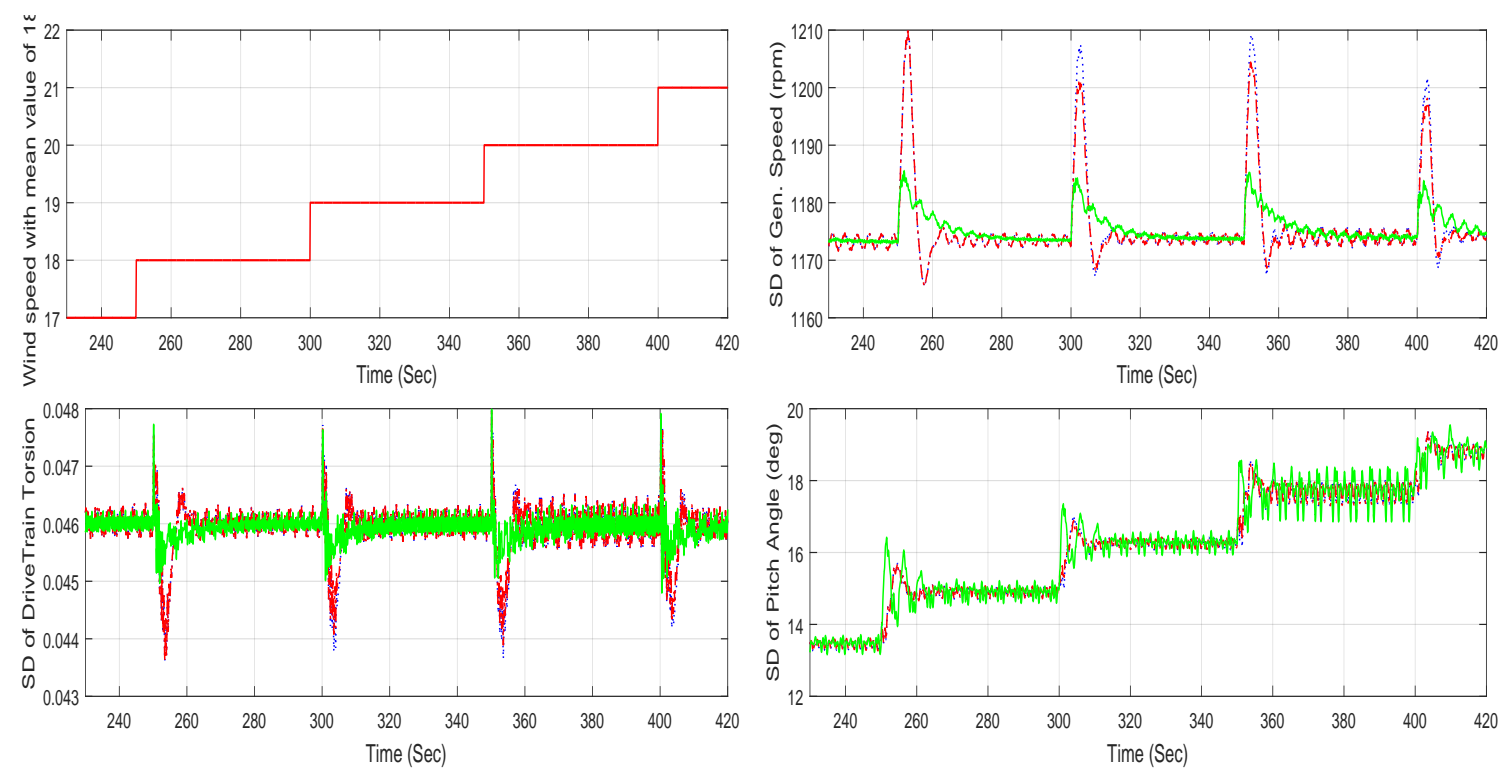

Figure 3. Step changine wind simulation results.

Blueline: Fixed Gain-CPC, Redline: LPV-CPC, Greenline: LPV-IPC
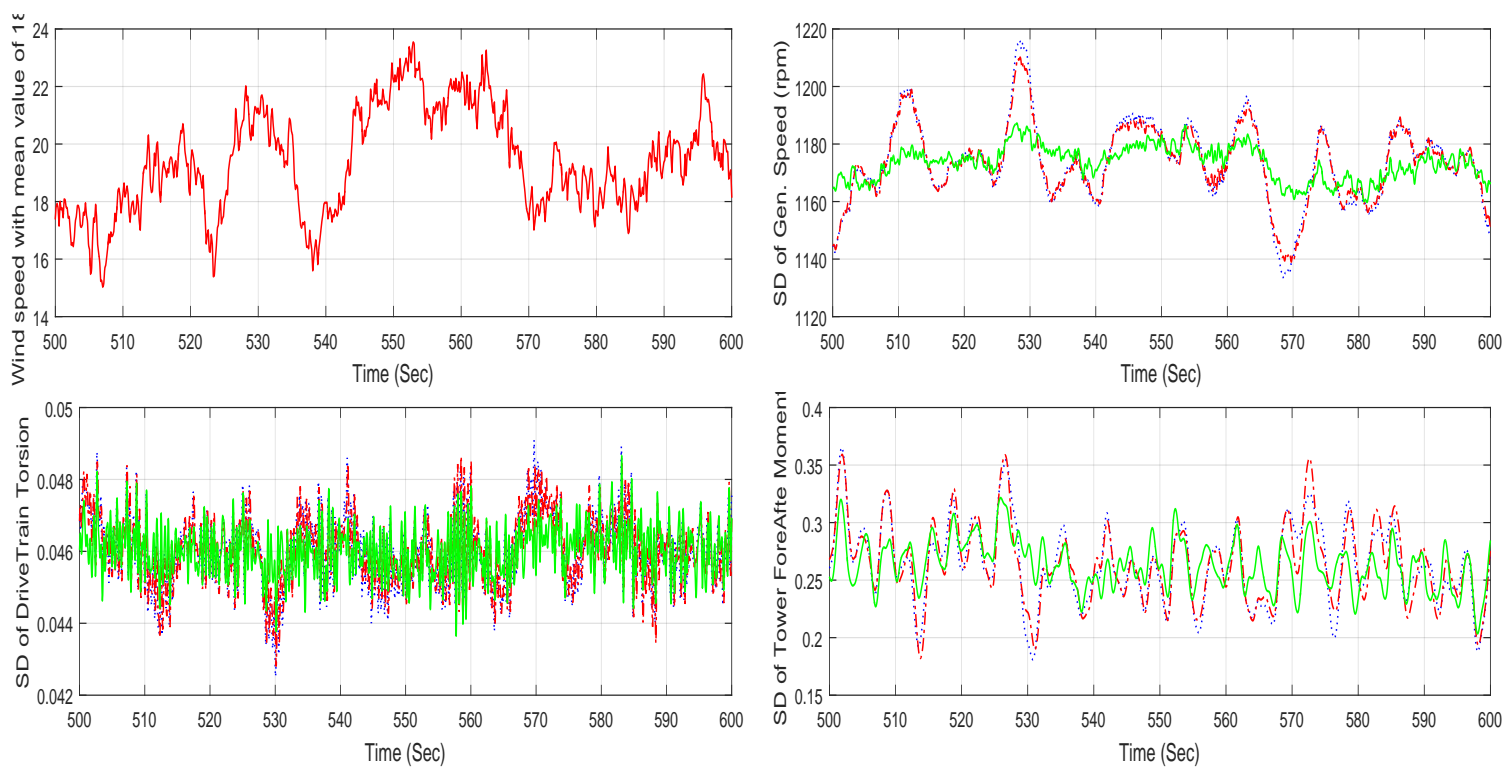

Figure 4. Turbulent wind simulation results.

Blueline: Fixed Gain-CPC, Redline: LPV-CPC, Greenline: LPV-IPC

Results of turbulent wind simulation are summarized in Table 1. It can be inferred from the results that percentage improvement in the standard deviation of the generator speed, drive-train torsion and tower moments are respectively $9 \%, 2 \%$ and $2 \%$ compared to fixed gain-CPC. Furthermore $60 \%, 39 \%$ and $29 \%$ percentage improvement in the standard deviation of the generator speed, drive-train torsion and tower moments are observed compared to fixed gain-CPC. However better performance can be achieved by the tuning of the multivariable controller at the operating points to do better mitigation to loads of components, regulation of output power and reduced pitching activity. 
Table 1. Standard Deviation of Parameters

\begin{tabular}{c|c|c|c}
\hline Parameter & Fixedgain-CPC & LPV-CPC & LPV-IPC \\
\hline \hline Gen. Speed $(\mathrm{rpm})$ & 14.22 & 12.95 & 5.73 \\
\hline Torsion $\left(\mathrm{x} 10^{-3} \mathrm{deg}\right)$ & 950 & 930 & 680 \\
\hline Tower Moment $(\mathrm{KNm})$ & 0.034 & 0.033 & 0.021 \\
\hline
\end{tabular}

\section{Conclusions}

This paper has presented a systematic method for designing a parametrized DOBC with IPC for full load operations of wind turbine. The proposed controller is based on the LPV design method that provides a smooth transition between two multivariable DOBC. Controllers are interpolated between the two operating point without any bump. It is tested with step changing wind and then switching between the controllers is checked by applying turbulent wind. Analysis of the simulation results shows that the proposed controller reduced the load of drive train, gearbox and tower moment in the presence of cyclic loads and better regulation to the produced power. It should be noted that model uncertainty are not directly handled in the design formulation. The performance can be increased by retuning of the controller with objectives to reduce tower oscillations, drive train torsion, mitigate periodic aerodynamic loads and individual pitch controller can also be accommodated in the controller design for the full load operation of wind turbine.

\section{References}

1. Anjun, Xia, et al. "Pitch control of large scale wind turbine based on expert PID control." Electronics, Communications and Control (ICECC), 2011 International Conference on. IEEE, 2011.

2. Yao, Xingjia, et al. "Pitch regulated LQG controller design for variable speed wind turbine." Mechatronics and Automation, 2009. ICMA 2009. International Conference on. IEEE, 2009.

3. Kalbat, Abdulrahman. "Linear Quadratic Gaussian (LQG) control of Wind turbines." Electric Power and Energy Conversion Systems (EPECS), 2013 3rd International Conference on. IEEE, 2013.

4. Imran, Raja M., D. M. Akbar Hussain, and Zhe Chen. "LQG controller design for pitch regulated variable speed wind turbine." Energy Conference (ENERGYCON), 2014 IEEE International. IEEE, 2014.

5. Zhang, Yunqian, et al. "Flicker mitigation strategy for a doubly fed induction generator by torque control." Renewable Power Generation, IET 8.2 (2014): 91-99.

6. Zhang, Yunqian, et al. "Flicker mitigation by individual pitch control of variable speed wind turbines with DFIG." Energy Conversion, IEEE Transactions on 29.1 (2014): 20-28.

7. Imran, Raja M., D. M. Akbar Hussain, and Mohsen Soltani. "DAC with LQR control design for pitch regulated variable speed wind turbine." Telecommunications Energy Conference (INTELEC), 2014 IEEE 36th International. IEEE, 2014.

8. Imran, Raja M., D. M. Akbar Hussain, and Mohsen Soltani. "DAC to mitigate the effect of periodic disturbances on drive train using collective pitch for variable speed wind turbine." Industrial Technology (ICIT), 2015 IEEE International Conference on. IEEE, 2015.

9. Johansson, Karl H., et al. "Multivariable controller tuning." American Control Conference, 1998. Proceedings of the 1998. Vol. 6. IEEE, 1998.

10. Njiri, Jackson G., Yan Liu, and Dirk Söffker. "Multivariable control of large variable-speed wind turbines for generator power regulation and load reduction." IFAC-PapersOnLine 48.1 (2015): 544-549.

11. Boukhezzar, Boubekeur, et al. "Multivariable control strategy for variable speed, variable pitch wind turbines." Renewable Energy 32.8 (2007): 1273-1287.

12. Geyler, M., and P. Caselitz. "Robust multivariable pitch control design for load reduction on large wind turbines." Journal of solar energy engineering 130.3 (2008): 031014.

13. Muhammad Nizam, Kamarudin, Md Rozali Sahazati, and Husain Abdul Rashid. "Observer-based output feedback control with linear quadratic performance." (2012). 
14. Chen, Wen-Hua, et al. "Disturbance observer-based control and related methods: An overview." IEEE Transaction on Industrial Electronics, Vol. 63, No. 2, February 2016,

15. Østergaard, Kasper Zinck, Jakob Stoustrup, and Per Brath. "Linear parameter varying control of wind turbines covering both partial load and full load conditions." International Journal of Robust and Nonlinear Control 19.1 (2009): 92-116.

16. Hangstrup, Mads E., et al. "Gain-scheduled control of a fossil-fired power plant boiler." Control Applications, 1999. Proceedings of the 1999 IEEE International Conference on. Vol. 2. IEEE, 1999.

17. Stoustrup, Jakob, and Mohammad Komareji. "A parameterization of observer-based controllers: Bumpless transfer by covariance interpolation." Proc. American Control Conf., St. Louis, MO, USA. 2009.

18. Niss, Michael Odgaard Kuch, et al. "A Youla-Kucera approach to gain-scheduling with application to wind turbine control." CCA/ISIC. 2009.

19. Østergaard, Kasper Zinck. Robust, gain-scheduled control of wind turbines. PhD diss., Automation and Control, Department of Electronic Systems, Aalborg University, 2008.

20. Bagherieh, Omid. "Gain-scheduling control of floating offshore wind turbines on barge platforms." Electronic Theses and Dissertations (ETDs) 2008+ (2013).

21. Shirazi, Farzad A., Karolos M. Grigoriadis, and Daniel Viassolo. "Wind turbine linear parameter varying control using fast code." ASME 2012 5th Annual Dynamic Systems and Control Conference joint with the JSME 2012 11th Motion and Vibration Conference. American Society of Mechanical Engineers, 2012.

22. $\mathrm{Bu}$, Yiming. Switching linear parameter-varying control of a variable-speed wind turbine. California State University, Long Beach, 2015.

23. Jonkman JM, Buhl Jr ML. "FAST user's guide". National Renewable Energy Laboratory, Golden, CO, Technical Report No. NREL/EL-500-38230. 2005 Aug.

24. Imran, Raja M, "Multivariable Control for Load Mitigation of Wind Turbine." PhD Thesis, Aalborg University, Esbjerg, Denmark (2016).

25. Imran, Raja M., D. M. Akbar Hussain, Mohsen Soltani and Raja M. Rafaq"Optimal Tuning of Multivariable Disturbance-Observer-Based Control for Flicker Mitigation Using IPC of Wind Turbine." Journal of IET Renewable Power Generation (2016).

26. Soltani, Mohsen Nourbakhsh, et al. "Estimation of rotor effective wind speed: A comparison." Control Systems Technology, IEEE Transactions on 21.4 (2013): 1155-1167.

27. Anderson, Brian DO, and John B. Moore. Optimal control: linear quadratic methods. Courier Corporation, 2007.

28. Pintea, Andreea, et al. "Optimal control of variable speed wind turbines." Control Automation (MED), 2011 19th Mediterranean Conference on. IEEE, 2011.

29. Butterfield S, Musial W, Scott G."Definition of a 5-MW reference wind turbine for offshore system development." (2009). 